\title{
Wi-fi fingerprint database construction using Chebyshev wavelet functions
}

\begin{abstract}
In this research we propose a new approach of constructing Wi-Fi fingerprints database using Chebyshev wavelet functions. Proposed algorithm combines the reference data collection procedure with path-loss prediction. Our algorithm requires only a few samples to be collected in a given region, and thus significantly reduces the calibration time. Next, the collected reference samples are used to construct area specific path-loss prediction function using Chebyshev wavelets. Once we have the path-loss function, we can predict the signal strength values at any point of the target area. One of the advantages of proposed algorithm is that it does not require any detailed information about target area, i.e. location of wireless access points, number of walls (floors, obstructions) between the transmitter and reference points and etc.
\end{abstract}

